\title{
UNIVERSITYOF BIRMINGHAM

\section{Point of Care Ultrasound Accurately Distinguishes Inflammatory from Noninflammatory Disease in Patients Presenting with Abdominal Pain and Diarrhea}

Novak, Kerri L; Jacob, Deepti; Kaplan, Gilaad; Boyce, Emma; Ghosh, Subrata; Ma, Irene; Lu, Cathy; Wilson, Stephanie; Panaccione, Remo

DOI:

10.1155/2016/4023065

License:

Creative Commons: Attribution (CC BY)

\section{Document Version}

Publisher's PDF, also known as Version of record

Citation for published version (Harvard):

Novak, KL, Jacob, D, Kaplan, G, Boyce, E, Ghosh, S, Ma, I, Lu, C, Wilson, S \& Panaccione, R 2016, 'Point of Care Ultrasound Accurately Distinguishes Inflammatory from Noninflammatory Disease in Patients Presenting with Abdominal Pain and Diarrhea', Canadian Journal of Gastroenterology and Hepatology, vol. 2016, 4023065. https://doi.org/10.1155/2016/4023065

Link to publication on Research at Birmingham portal

\footnotetext{
General rights

Unless a licence is specified above, all rights (including copyright and moral rights) in this document are retained by the authors and/or the copyright holders. The express permission of the copyright holder must be obtained for any use of this material other than for purposes permitted by law.

- Users may freely distribute the URL that is used to identify this publication.

- Users may download and/or print one copy of the publication from the University of Birmingham research portal for the purpose of private study or non-commercial research.

- User may use extracts from the document in line with the concept of 'fair dealing' under the Copyright, Designs and Patents Act 1988 (?)

- Users may not further distribute the material nor use it for the purposes of commercial gain.

Where a licence is displayed above, please note the terms and conditions of the licence govern your use of this document.

When citing, please reference the published version.

Take down policy

While the University of Birmingham exercises care and attention in making items available there are rare occasions when an item has been uploaded in error or has been deemed to be commercially or otherwise sensitive.

If you believe that this is the case for this document, please contact UBIRA@lists.bham.ac.uk providing details and we will remove access to the work immediately and investigate.
} 


\title{
Point of Care Ultrasound Accurately Distinguishes Inflammatory from Noninflammatory Disease in Patients Presenting with Abdominal Pain and Diarrhea
}

\author{
Kerri L. Novak, ${ }^{1}$ Deepti Jacob, ${ }^{1}$ Gilaad G. Kaplan, ${ }^{1,2}$ Emma Boyce, ${ }^{1}$ Subrata Ghosh, ${ }^{1}$ \\ Irene $\mathrm{Ma}^{3}{ }^{3}$ Cathy Lu, ${ }^{4}$ Stephanie Wilson, ${ }^{5}$ and Remo Panaccione ${ }^{1}$ \\ ${ }^{1}$ Inflammatory Bowel Disease Clinic, Division of Gastroenterology and Hepatology, University of Calgary, Calgary, \\ AB, Canada T2N 2 T9 \\ ${ }^{2}$ Department of Community Health Sciences, Calgary, AB, Canada T2N 2T9 \\ ${ }^{3}$ Division of General Internal Medicine, Calgary, AB, Canada T2N 2T9 \\ ${ }^{4}$ Division of Gastroenterology, University of Alberta, Edmonton, AB, Canada T2N 2T9 \\ ${ }^{5}$ Diagnostic Imaging, University of Calgary, Calgary, AB, Canada T2N 2T9
}

Correspondence should be addressed to Kerri L. Novak; knovak@ucalgary.ca

Received 24 August 2015; Accepted 29 September 2015

Copyright (C) 2016 Kerri L. Novak et al. This is an open access article distributed under the Creative Commons Attribution License, which permits unrestricted use, distribution, and reproduction in any medium, provided the original work is properly cited.

Background. Approaches to distinguish inflammatory bowel disease (IBD) from noninflammatory disease that are noninvasive, accurate, and readily available are desirable. Such approaches may decrease time to diagnosis and better utilize limited endoscopic resources. The aim of this study was to evaluate the diagnostic accuracy for gastroenterologist performed point of care ultrasound (POCUS) in the detection of luminal inflammation relative to gold standard ileocolonoscopy. Methods. A prospective, single-center study was conducted on convenience sample of patients presenting with symptoms of diarrhea and/or abdominal pain. Patients were offered POCUS prior to having ileocolonoscopy. Sensitivity, specificity, positive predictive value (PPV), and negative predictive value (NPV) with 95\% confidence intervals (CI), as well as likelihood ratios, were calculated. Results. Fifty-eight patients were included in this study. The overall sensitivity, specificity, PPV, and NPV were $80 \%, 97.8 \%, 88.9 \%$, and $95.7 \%$, respectively, with positive and negative likelihood ratios (LR) of 36.8 and 0.20 . Conclusion. POCUS can accurately be performed at the bedside to detect transmural inflammation of the intestine. This noninvasive approach may serve to expedite diagnosis, improve allocation of endoscopic resources, and facilitate initiation of appropriate medical therapy.

\section{Introduction}

Irritable bowel syndrome (IBS) is a functional gastrointestinal (GI) disorder, characterized by chronic abdominal pain and altered bowel habit, with a benign natural history. With a worldwide prevalence approximating $10-15 \%$, it is a common disorder and one of the most frequent sources of referral to gastroenterology [1]. The potential economic burden of IBS is significant, and some patients have long-term persistence of symptoms with need for more chronic management strategies [1-3]. In contrast, the incidence of the inflammatory bowel disease (IBD) in North America is only a fraction of IBS [4].

A subset of patients with IBS have diarrhea and abdominal pain as predominant symptoms, presenting similarly to those with inflammatory bowel disease (IBD), thus making the clinical distinction challenging based on symptoms alone $[5,6]$. Timely differentiation of IBD from IBS is critical because delay in diagnosis of IBD can increase the risk of developing complications, hospitalization for management, and surgery [7].

Clinical indices used to accurately identify inflammation in patients with symptoms exist but are not discerning for IBD and have not been incorporated into routine diagnostic investigation [8]. Laboratory markers are also not always consistent predictors of inflammation to exclude IBD [9]. For example, fecal inflammatory markers may exclude inflammation present in the colon but are not always reliable for small bowel disease common in Crohn's disease [10]. 
Endoscopy represents the gold standard diagnostic modality to investigate symptoms that suggest inflammation. Endoscopic resources are limited, however, and the number of patients with abdominal symptoms diagnosed at endoscopy with IBD is low [5]. In addition, current guidelines suggest that many patients should be managed conservatively with symptom-based diagnosis, given the low yield of traditional means to detect organic disease $[11,12]$. Alternate, noninvasive diagnostic strategies such as computed tomography (CT) and magnetic resonance imaging (MRI) are not routinely recommended for the investigation of suspected IBS, yet these strategies are increasingly used [13]. Screening with these modalities for inflammation or other worrisome etiology in IBS should be limited, given the concern over radiation exposure with CT and the high cost and limited access to MRI [13]. Ultrasound (US) is a noninvasive, accurate, safe, effective, and easily accessible modality. It can be performed in clinic by gastroenterologists as an extension of the physical examination [14].

The use of point of care ultrasound (POCUS) for noncardiac purposes in medicine by nonradiologists has increased significantly in the last decade [15]. Focused examination performed at the bedside during routine clinical assessment may be used to answer binary diagnostic questions: "does this patient have free fluid in the abdomen?" [16]. Although widely implemented as an integral part of routine evaluation for patients with established IBD in many parts of Europe, it is not widely used as a diagnostic tool by gastroenterologists in North America. The aim of this prospective study of patients investigated for symptoms to exclude organic disease was to evaluate the diagnostic accuracy of gastroenterologist performed POCUS in excluding luminal inflammation relative to gold standard ileocolonoscopy. This tool may be used in the future to help guide the appropriate use of diagnostic endoscopy.

\section{Materials and Methods}

2.1. Patient Population and Study Design. Consecutive patients presenting for assessment of symptoms including abdominal pain and diarrhea suspicious for IBD to the University of Calgary GI clinic were selected for this pilot study from January 1, 2013, to June 1, 2015. Patients with significant abdominal obesity (BMI > 40), pregnancy, liver disease with ascites, or conditions of previously known luminal inflammation were excluded. Informed consent was obtained prior to the examination and the study was approved by the Conjoint Health Research Ethics Board guidelines.

2.2. Clinical Assessment and POCUS Examination. All patients underwent a physical examination as per standard of care. Laboratory inflammatory markers including Creactive protein (CRP) were recorded where available, within 2 months of POCUS. Fecal calprotectin was not available at the time of the study at this center. A gastroenterologist (KN), with formal training in US of the GI tract and 3-year experience in the modality, conducted a focused luminal POCUS examination. A standardized approach was used, conducting the examination from the left lower quadrant, examining the colon from the rectum to the cecum, with examination of the rectum, sigmoid, descending, transverse, and ascending colon, and cecum. The terminal ileum was then evaluated, followed by systematic four-quadrant examination to include the remaining small bowel. All exams were completed using a Phillips IU22 US machine utilizing a range of transducers, including high frequency curved (4-9 $\mathrm{mHz})$ and linear probes $(12-15 \mathrm{mHz})$.

Presence of inflammatory activity was documented in binary fashion ("active"/inactive"). Inflammation was deemed to be present, as per standard sonographic assessment, if there was increased bowel wall thickness ( $>3 \mathrm{~mm}$ for small bowel, $>4 \mathrm{~mm}$ for large bowel) and the presence of any additional established indicators of inflammation: mesenteric inflammatory fat, lymph nodes, and hyperemia or blood flow as detected on color Doppler imaging [17]. The anatomic site(s) of disease was also recorded. In addition, complications including luminal stenoses, penetrating complications or fistulas, perianal disease, and intra-abdominal abscesses were described and recorded if present. Finally, commentary was made regarding echo-stratification or preservation of wall layers as well as the overall quality of the examination (adequate/inadequate).

Patients with normal blood work, negative endoscopy, and histology and who met Rome III criteria for IBS were identified as having IBS (diarrhea predominant) [18]. Those with normal endoscopic features were diagnosed with microscopic colitis, if confirmation was made histologically [19]. Inflammatory bowel disease (IBD) was diagnosed/confirmed where both endoscopic and histologic markers were present; in these cases anatomic locations and patterns were recorded. The gold standard comparator was endoscopy alone for all studies. Finally, alternate etiologies including segmental colitis associated with diverticula, infectious, or ischemic colitis were also recorded.

2.3. Endoscopic and Histologic Examination. All patients underwent ileocolonoscopy after POCUS. The time interval to routine or expedited endoscopy, determined based on clinical suspicion of disease, was recorded. Extent of the endoscopy was recorded including intubation of the terminal ileum or lack of as well as the presence of disease activity, which was recorded in a binary fashion (present/absent), along with documentation of the etiology of the inflammation (confirmation of Crohn's disease or ulcerative colitis or other suspected etiology) and, if present, the anatomic location. Where clinically indicated, biopsies were taken in both the small and large bowel and results (presence/absence of inflammation) were recorded. If biopsies were taken as part of the standard evaluation, this too was recorded as confirmation of organic disease or its absence.

2.4. Patient Satisfaction. A subset of patients (14/58) completed a 12-question patient satisfaction questionnaire, with graded responses following the POCUS examination in clinic. The questionnaire evaluated patient satisfaction with the overall experience, patient perception of the value of 
TABle 1: Patient demographic and laboratory investigation data (change title).

\begin{tabular}{lc}
\hline & $n(\%)$ \\
\hline Gender & $19(33)$ \\
$\quad$ Male & $39(67)$ \\
$\quad$ Female & $32.8(17.8-72.4)$ \\
Median age (years) & $3.4(0.6-36.7)$ \\
Median CRP (mmL, range) $(n=31)^{*}$ & $39.7(0-127)$ \\
$\begin{array}{l}\text { Median time between POCUS and CRP } \\
\text { (days) }\end{array}$ & $30(0-149)$ \\
$\begin{array}{l}\text { Median time from POCUS to endoscopy } \\
\text { (days, range) }\end{array}$ & $56(96.5)$ \\
Adequate POCUS exam quality & $56(96.6)$ \\
\hline TI intubation on endoscopy
\end{tabular}

* Only a subset had CRP measured that is the $n=31$.

POCUS, and the willingness to incorporate POCUS as an extension of the physical examination in clinic.

2.5. Statistical Analysis. Demographic data and ultrasound quality were evaluated using descriptive statistics. Further analysis was aimed at evaluating the ability of POCUS to detect inflammation in symptomatic patients compared with standard colonoscopy; again, colonoscopy was considered the gold standard. Sensitivity, specificity, positive predictive value (PPV), negative predictive value (NPV), and the positive and negative likelihood ratios (LR) with $95 \%$ confidence intervals (CI) were calculated for the overall POCUS score, compared to endoscopy only. To further evaluate POCUS, the above-mentioned statistics were stratified for segmental analysis, either ileum or colon. Accuracy of the detection of disease location, if present, was also evaluated. In cases where the ileum was not seen in endoscopic examinations, correlations were made between the endoscopic and US segments of the colon alone. Finally, the ultrasound examination quality was measured descriptively. Histology was reported; however, it was not used as the gold standard.

\section{Results}

A total of 58 patients presenting to the clinic with symptoms were included. Demographic details are presented in Table 1. Two patients with a high BMI ( $>35$ ) contributed to poor exam quality; however, the bowel was visualized; thus, they were included in the analysis. The remaining 56/58 (96\%) examinations were of good quality. Endoscopies were performed following POCUS with a median of 30 days and all were included in the analysis. All but 2 examinations intubated the terminal ileum (Table 1).

Inflammatory activity was detected sonographically (positive study) in $9 / 58$ (15.5\%) ranging from mild to severe inflammation (Tables 2 and 3). Mesenteric lymph nodes and increased bowel wall thickness were the most common US parameters identified and were seen in all patients with US activity (9/9 100\%) (Figure 1). The only complication identified was a stricture, present in $1 / 9$ cases $(11 \%)$, characterized
TABLE 2: Descriptive ultrasonographic and endoscopic data.

\begin{tabular}{lc}
\hline & Ultrasound \\
& $N(\%)$ \\
\hline Active inflammation on US & $9 / 58(15.5)$ \\
Increased bowel wall thickness & $9 / 9(100)$ \\
Lymph nodes & $9(100)$ \\
Inflammatory fat & $7(78)$ \\
Hyperemia & $3(33)$ \\
Preserved wall layers & $10(100)$ \\
Complications ${ }^{+}$ & $1(11 \%)$ \\
Active site on endoscopy $(n=10)$ & \\
Ileum & $4(40 \%)$ \\
Colon & $5(50)$ \\
Ileocolonic & $1(10 \%)$ \\
\hline${ }^{+}$Complications identified include any of abscess, stricture, phlegmon, or \\
inflammatory mass.
\end{tabular}

TABLE 3: A $2 \times 2$ table for ultrasound compared to gold standard endoscopy.

\begin{tabular}{lcc}
\hline & Positive endoscopy & Negative endoscopy \\
\hline Positive US & 8 & 2 \\
Negative US & 1 & 47 \\
\hline
\end{tabular}

TABLE 4: Sensitivity, specificity, PPV, and NPV of POCUS relative to endoscopy.

\begin{tabular}{lccc}
\hline & Overall & Ileum & Colon \\
\hline Sensitivity\% & 80.0 & 100.0 & 60.0 \\
(CI\%) & $(44.4-97.5)$ & $(47.8-100.0)$ & $(914.7-94.7)$ \\
Specificity\% & 97.8 & 98.2 & 100.0 \\
(CI\%) & $(88.5-99.9)$ & $(90.45-99.9)$ & $(93.3-100.0)$ \\
PPV\% & 88.9 & 83.3 & 100.0 \\
(CI\%) & $(51.7-99.7)$ & $(35.9-99.6)$ & $(29.2-100.0)$ \\
NPV\% & 95.7 & 100.0 & 96.36 \\
(CI\%) & $(85.5-99.5)$ & $(93.5-100.0)$ & $(87.5-99.6)$ \\
Positive LR & 36.8 & 56.0 & - \\
(CI\%) & $(5.2-262.1)$ & $(8.0-390.6)$ & $(-)$ \\
Negative LR & 0.20 & - & 0.40 \\
(CI\%) & $(0.06-0.71)$ & $(-)$ & $(0.14-1.17)$ \\
\hline
\end{tabular}

by fixed luminal apposition and narrowing, with evidence of proximal small bowel dilation. Ten (10/58 or 17\%) patients had inflammation confirmed on endoscopy (Tables 3 and 4). Most patients (93\%) had biopsies and pathology completed, and all 10 had inflammation confirmed histologically. Of these, 4/10 (40\%) had ileal CD (Figure 1), 1 with ileocolonic distribution of inflammation, 5 had colonic inflammation (1 with a new diagnosis of ulcerative colitis, 1 with segmental diverticular inflammation, and the remaining 3 had patchy, mild colonic inflammation consistent with colonic CD). There was one false-positive US exam, with 2 false-negative findings (see $2 \times 2$ data in Table 3). In addition, 9 patients with normal colons both sonographically and endoscopically 


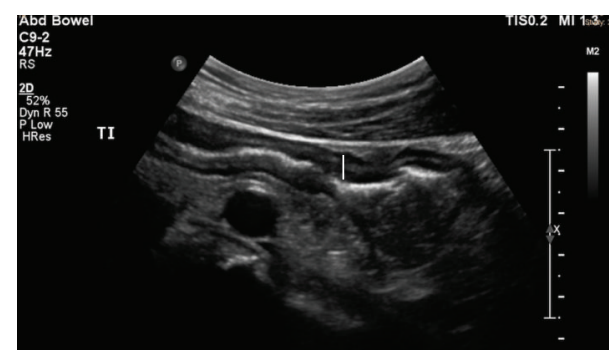

FIGURE 1: First suggestion of terminal ileal Crohn's disease identified on sonography, with thickened distal ileum running over the hypoechoic iliac artery. The white line marks the thickened ileal wall with echogenic or white air in the lumen.

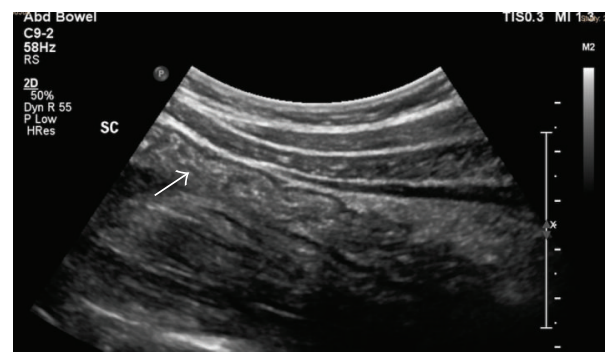

FIGURE 2: Normal sigmoid colon with normal haustral folds (white arrow).

revealed microscopic colitis on pathology. One patient exhibited no evidence of disease endoscopically nor on US but had mild inflammatory activity identified histologically in the ileum, suggesting possibly early IBD. All other cases confirmed diarrhea-predominant IBS (38/58 65.5\%) (Figure 2). There were no cases of ulcerative colitis identified in this study. In those patients with a positive POCUS, endoscopic exams occurred with shorter intervals with a median of days. The shortest wait time for endoscopy after US was endoscopy on the same day of the US exam.

POCUS exhibited a PPV of $88.9 \%$ (95\% CI: $51.75-$ 99.72) and a NPV of $95.7 \%$ (95\% CI: 85.5-99.5). The overall sensitivity and specificity of POCUS were $80 \%$ (95\% CI: $44.4-$ 97.5) and 97.8\% (95\% CI: 88.5-99.9), respectively, using a cutoff for bowel wall thickness of $3 \mathrm{~mm}$ for the ileum and $4 \mathrm{~mm}$ for the colon, with positive and negative LRs of 36.8 and 0.20 (Table 3 ). When the results were analyzed by segment, comparing colonic disease and ileal disease separately, ultrasound detection of ileal disease exhibited higher sensitivity compared to the colon compared to gold standard endoscopy (Table 3).

Of the 10 patients with confirmed inflammatory activity on endoscopy, all had CRP (7/10 within 1 month) completed, and 5 were elevated while the other 5 were within normal range. The median CRP value for these 10 patients was $6.9 \mathrm{mg} / \mathrm{L}$. In those with elevated CRP, none of the US examination, confirmed endoscopically, revealed severe disease while all those 3 cases with moderate to severe sonographic disease activity had CRP within normal range, measured within $40 \mathrm{~d}$ of the US.
All 14 patients who completed questionnaires described the preparation for US as "easy" and reported an increased understanding of their disease and disease location following the exam. All of these patients believed POCUS had value in guiding disease management and found the increased clinic time acceptable and beneficial to their health.

\section{Discussion}

To our knowledge, this is the first prospective study to evaluate the accuracy of gastroenterologist-performed POCUS in excluding inflammation in symptomatic patients compared to colonoscopy in North America. The accuracy established here is similar to previous published data [20-22]. There is a significant need for noninvasive, safe, resource-conscious modalities of accurately detecting inflammation in symptomatic patients, as symptoms alone do not accurately reflect inflammatory disease activity and incorrect diagnosis can delay appropriate management of IBD $[23,24]$. Ultrasound has been shown to significantly impact clinical decisions, when used at the bedside [25]. Symptoms of diarrhea and abdominal pain are common as manifestations of irritable bowel syndrome, and diarrhea is a symptom that often drives patient visits to primary care $[1,21]$. Commonly used noninvasive markers such as C-reactive protein (CRP) may be falsely negative and fecal calprotectin has variable availability and may have a lower accuracy in detecting small bowel inflammation $[9,26]$.

The rate of pathologically proven histologic inflammation in this study, either microscopic colitis or inflammatory bowel disease, was 20/58 (34\%). This figure is much higher than that quoted for larger populations of patients with IBS including all subtypes of IBD. Given their symptoms, this population was identified as "high risk" and in need of ileocolonoscopy as part of standard of care investigation and the high pathology rate likely reflects some bias or overrepresentation of pathology, given the convenience sampling from the "high-risk IBD" clinic. However, gold standard evaluation with endoscopy is not always indicated in IBS patients, as it is resource intensive and carries the associated risks of an invasive examination (including patient tolerance) $[12,22]$. Tools to support indication for endoscopy would be helpful, in addition to the timing/urgency of endoscopy. In this study, $83 \%(48 / 58)$ of patients had no evidence of inflammation on POCUS or endoscopy, suggesting that ultrasound may obviate the need for endoscopy in select patients, especially in combination with a low CRP, as recent evidence suggests that CRP $<1.0$ makes the probability of IBD very low [9]. Clinical factors suggesting organic etiology including $\mathrm{MC}$ may also be useful, such as night time stooling, weight loss, and age greater than 50 years [27]. In this study, CRP was not suggestive of more severe disease either sonographically or endoscopically, illustrating, even in this small sample, limitations with using CRP as a guide for the need for endoscopy. Given current wait-times for nonurgent endoscopy, inappropriate delays may result as a consequence of false-negative results of currently available tests [24]. Thus, a widely available, accurate set of clinical tools that would include noninvasive US may guide the appropriate use of diagnostic 
endoscopy. This has been demonstrated here with the high likelihood ratio of US, suggesting a very high probability of confirming IBD with a positive scan [28]. This may allow for confident expedition of urgent diagnostic assessment and early appropriate therapy. Here, abnormal US findings resulted in expedited endoscopy with median time to diagnosis of 26 days in those with active IBD on sonography, compared to a median time to endoscopy of 30 days for the study population.

The accuracy of detecting disease in the terminal ileum on cross-sectional imaging tends to be greater compared to the colon, as was exhibited in this study on US [14]. The sensitivity and specificity for detecting ileal disease was higher compared to colonic disease (Table 4). Both cases (2/10) missed on US were mild endoscopically, possibly not yet exhibiting cross-sectional disease activity [29]. Regardless of imaging modality, mild disease is difficult to detect, given the absence of transmural involvement, as early disease involves the mucosa and this is also reflected here, as there were 2 false negatives versus only 1 false positive (Table 3 ). This circumstance may be best served by the addition of fecal calprotectin, valuable for detecting inflammation in this context [9]. Although a normal US may not obviate the need for endoscopic confirmation, it was however reassuring and suggested a need for a less urgent endoscopy as any disease missed was most likely relatively mild. There were no cases where the distal terminal ileum was normal on endoscopy and disease was isolated to the proximal small bowel, as has been reported in up to $30 \%$ of cases with small bowel involvement [30]; however, in 2 patients with both US and endoscopic activity (true positives), there was an estimation between 25 and $30 \mathrm{~cm}$ of active disease proximal to that region examined endoscopically. Thus, US also provided insight into the length or burden of the disease [31]. One case exhibited negative US and negative endoscopy, yet mild inflammatory activity on pathology. This may indicate early inflammatory bowel disease, an important etiology to detect early in order to alter the natural disease history. This again highlights the need for concomitant clinical tools such as stool and serum-based inflammatory markers, combined with clinical factors, to direct appropriateness of endoscopic evaluation and biopsies.

Nine patients with both negative US and endoscopy had microscopic colitis (MC) confirmed histologically. Five of these patients (55\%) exhibited extensive small bowel fluid filled loops, a novel finding suggesting possible pathology of the small bowel as well (Figure 2). This requires investigation and confirmation with larger numbers. Small bowel involvement in MC is not well understood and may be missed on endoscopy because the small bowel may not be extensively visualized or biopsied. Thus, POCUS may also contribute significant understanding of anatomy and function in regions of the bowel not seen endoscopically.

Image quality has been suggested to be more variable in US compared to MR or CT, potentially limiting US in some populations, specifically in those with high BMI (>35) with increased abdominal wall adiposity. Only 2 patients in this study had poor quality examinations due to high BMI; however, the US examinations were still deemed sufficient. There are no currently existing training guidelines for gastroenterologists to guide minimal requirement for competency in luminal sonography in Canada, given the evolution of this emerging modality in the field. However, it is a standard part of training in many European countries including Germany and Italy, where at least 100 examinations are recommended [32]. Inexperienced sonographers have also shown to have significant ability to accurately detect disease [33].

Patient engagement and satisfaction are important components of establishing rapport and trust and are crucial for long-term success in the management of both chronic functional disorders and inflammatory conditions. US improved patient understanding and was well tolerated and was generally preferred over invasive endoscopy. This tool may prove to be important in facilitating understanding and reassurance for patients at diagnosis and is a radiation-free and relatively inexpensive modality that can be used serially for disease monitoring in established IBD. The sample reflecting patient satisfaction was however small, in this early pilot study.

There are a number of limitations in this study, as it is small and is limited to one academic center with one sonographer performing bedside US examinations. These limitations should of course be kept in mind when considering the generalizability of this study to other centers. This is, however, a novel modality in gastroenterology, and training guidelines along with reporting and quality frameworks must be established. In addition, this preliminary analysis is underpowered; therefore, a single error, false-positive or negative, will significantly adversely impact the sensitivity and specificity in this small sample. Thus, prospectively collected larger studies are necessary to understand accuracy of the modality with and without additional clinical factors such as clinical predictors and inflammatory markers.

The time interval between US and endoscopy was variable with few endoscopic examinations completed more than 3 months after the POCUS. This likely reflects real life practice, however, as these patients were clinically suspected to have IBS and investigation for most of them was triaged as nonurgent. There were no medical interventions initiated for any patients; therefore, no structural changes should result between imaging and endoscopic evaluations. Finally, the sonographer in this study was also performing ileocolonoscopy, not blinded to the US results, which may bias the commentary on endoscopy. In order to minimize this bias, nearly all (94\%) had biopsies taken to definitively exclude unrecognized inflammation.

\section{Conclusion}

Point of care US is a safe, noninvasive means of accurately detecting inflammation in patients being investigated for symptoms of diarrhea and abdominal pain. It is timely, as it occurs at the bedside during clinical assessment. It is well accepted by patients and can be employed to further direct definitive diagnosis and management and possibly obviate the need for endoscopic evaluation in certain cases. Given the high positive likelihood ratio with a positive exam, it can be effectively used as a triage tool to expedite endoscopy. 


\section{Competing Interests}

The authors declare that they have no competing interests.

\section{References}

[1] Y. A. Saito, P. Schoenfeld, and G. R. Locke III, “The epidemiology of irritable bowel syndrome in North America: a systematic review," The American Journal of Gastroenterology, vol. 97, no. 8, pp. 1910-1915, 2002.

[2] F. Creed, J. Ratcliffe, L. Fernandez et al., "Health-related quality of life and health care costs in severe, refractory irritable bowel syndrome," Annals of Internal Medicine, vol. 134, no. 9, part 2, pp. 860-868, 2001.

[3] N. Agarwal and B. M. R. Spiegel, "The effect of irritable bowel syndrome on health-related quality of life and health care expenditures," Gastroenterology Clinics of North America, vol. 40, no. 1, pp. 11-19, 2011.

[4] N. A. Molodecky, I. S. Soon, D. M. Rabi et al., "Increasing incidence and prevalence of the inflammatory bowel diseases with time, based on systematic review," Gastroenterology, vol. 142, no. 1, pp. 46.e42-54.e42, 2012.

[5] C. Canavan, T. Card, and J. West, "The incidence of other gastroenterological disease following diagnosis of irritable bowel syndrome in the UK: a cohort study," PLoS ONE, vol. 9, no. 9, Article ID e106478, 2014.

[6] P. Patel, P. Bercik, D. G. Morgan et al., "Prevalence of organic disease at colonoscopy in patients with symptoms compatible with irritable bowel syndrome: cross-sectional survey," Scandinavian Journal of Gastroenterology, vol. 50, no. 7, pp. 816-823, 2015.

[7] A. D. Frolkis, J. Dykeman, M. E. Negrón et al., "Risk of surgery for inflammatory bowel diseases has decreased over time: a systematic review and meta-analysis of population-based studies," Gastroenterology, vol. 145, no. 5, pp. 996-1006, 2013.

[8] C. Lahiff, P. Safaie, A. Awais et al., "The Crohn's Disease Activity Index (CDAI) is similarly elevated in patients with Crohn's disease and in patients with irritable bowel syndrome," Alimentary Pharmacology and Therapeutics, vol. 37, no. 8, pp. 786-794, 2013.

[9] S. B. Menees, C. Powell, J. Kurlander, A. Goel, and W. D. Chey, "A meta-analysis of the utility of C-reactive protein, erythrocyte sedimentation rate, fecal calprotectin, and fecal lactoferrin to exclude inflammatory bowel disease in adults with IBS," The American Journal of Gastroenterology, vol. 110, no. 3, pp. 444454, 2015.

[10] K. B. Gecse, J. F. Brandse, S. van Wilpe et al., "Impact of disease location on fecal calprotectin levels in Crohn's disease," Scandinavian Journal of Gastroenterology, vol. 50, no. 7, pp. 841-847, 2015.

[11] J.-R. Malagelada, "A symptom-based approach to making a positive diagnosis of irritable bowel syndrome with constipation," International Journal of Clinical Practice, vol. 60, no. 1, pp. 57-63, 2006.

[12] D. L. Dumitrascu, "Making a positive diagnosis of irritable bowel syndrome," Journal of Clinical Gastroenterology, vol. 45, supplement 2, pp. S82-S85, 2011.

[13] O. J. O'Connor, S. E. McSweeney, S. McWilliams et al., "Role of radiologic imaging in irritable bowel syndrome: evidence-based review," Radiology, vol. 262, no. 2, pp. 485-494, 2012.

[14] F. Parente, S. Greco, M. Molteni et al., "Role of early ultrasound in detecting inflammatory intestinal disorders and identifying their anatomical location within the bowel," Alimentary Pharmacology and Therapeutics, vol. 18, no. 10, pp. 1009-1016, 2003.

[15] L. Zieleskiewicz, L. Muller, K. Lakhal et al., "Point-of-care ultrasound in intensive care units: assessment of 1073 procedures in a multicentric, prospective, observational study," Intensive Care Medicine, vol. 41, no. 9, pp. 1638-1647, 2015.

[16] S. Adhikari, D. Morrison, M. Lyon, W. Zeger, and A. Krueger, "Utility of point-of-care biliary ultrasound in the evaluation of emergency patients with isolated acute non-traumatic epigastric pain," Internal and Emergency Medicine, vol. 9, no. 5, pp. 583-587, 2014.

[17] E. J. C. Sturm, L. P. J. Cobben, M. A. C. Meijssen, S. D. J. van der Werf, and J. B. C. M. Puylaert, "Detection of ileocecal Crohn's disease using ultrasound as the primary imaging modality," European Radiology, vol. 14, no. 5, pp. 778-782, 2004.

[18] A. C. Ford, P. Bercik, D. G. Morgan, C. Bolino, M. I. PintosSanchez, and P. Moayyedi, "Validation of the Rome III criteria for the diagnosis of irritable bowel syndrome in secondary care," Gastroenterology, vol. 145, no. 6, pp. 1262.el-1270.el, 2013.

[19] J. Tong, Q. Zheng, C. Zhang, R. Lo, J. Shen, and Z. Ran, "Incidence, prevalence, and temporal trends of microscopic colitis: a systematic review and meta-analysis," The American Journal of Gastroenterology, vol. 110, no. 2, pp. 265-276, 2015.

[20] F. Parente, S. Greco, M. Molteni et al., "Role of early ultrasound in detecting inflammatory intestinal disorders and identifying their anatomical location within the bowel," Alimentary Pharmacology \& Therapeutics, vol. 18, no. 10, pp. 1009-1016, 2003.

[21] N. Moreno, T. Ripollés, J. M. Paredes et al., "Usefulness of abdominal ultrasonography in the analysis of endoscopic activity in patients with Crohn's disease: changes following treatment with immunomodulators and/or anti-TNF antibodies," Journal of Crohn's and Colitis, vol. 8, no. 9, pp. 1079-1087, 2014.

[22] J. Panés, R. Bouzas, M. Chaparro et al., "Systematic review: the use of ultrasonography, computed tomography and magnetic resonance imaging for the diagnosis, assessment of activity and abdominal complications of Crohn's disease," Alimentary Pharmacology \& Therapeutics, vol. 34, no. 2, pp. 125-145, 2011.

[23] R. Sood, D. J. Gracie, G. R. Law, and A. C. Ford, "Systematic review with meta-analysis: the accuracy of diagnosing irritable bowel syndrome with symptoms, biomarkers and/or psychological markers," Alimentary Pharmacology \& Therapeutics, vol. 42, no. 5, pp. 491-503, 2015.

[24] S. M. Barratt, J. S. Leeds, K. Robinson, A. J. Lobo, M. E. McAlindon, and D. S. Sanders, "Prodromal irritable bowel syndrome may be responsible for delays in diagnosis in patients presenting with unrecognized Crohn's disease and celiac disease, but not ulcerative colitis," Digestive Diseases and Sciences, vol. 56, no. 11, pp. 3270-3275, 2011.

[25] K. Novak, D. Tanyingoh, F. Petersen et al., "Clinic-based point of care transabdominal ultrasound for monitoring Crohn's disease: impact on clinical decision making," Journal of Crohn's and Colitis, vol. 9, no. 9, pp. 795-801, 2015.

[26] A. Lasson, H. Strid, L. Öhman et al., "Fecal calprotectin one year after ileocaecal resection for Crohn's disease-a comparison with findings at ileocolonoscopy," Journal of Crohn's and Colitis, vol. 8, no. 8, pp. 789-795, 2014.

[27] G. Macaigne, P. Lahmek, C. Locher et al., "Microscopic colitis or functional bowel disease with diarrhea: a French prospective multicenter study," The American Journal of Gastroenterology, vol. 109, no. 9, pp. 1461-1470, 2014.

[28] S. McGee, "Simplifying likelihood ratios," Journal of General Internal Medicine, vol. 17, no. 8, pp. 647-650, 2002. 
[29] J. M. Paredes, T. Ripollés, X. Cortés et al., "Contrast-enhanced ultrasonography: usefulness in the assessment of postoperative recurrence of Crohn's disease," Journal of Crohn's and Colitis, vol. 7, no. 3, pp. 192-201, 2013.

[30] S. Samuel, D. H. Bruining, E. V. Loftus Jr. et al., "Endoscopic skipping of the distal terminal ileum in Crohn's disease can lead to negative results from ileocolonoscopy," Clinical Gastroenterology and Hepatology, vol. 10, no. 11, pp. 1253-1259, 2012.

[31] R. Wilkens, S. R. Wilson, K. L. Novak, and E. Lebeuf-Taylor, "Impact of intestinal ultrasound on classification and management of Crohn's disease patients with inconclusive colonoscopy," Canadian Journal of Gastroenterology \& Hepatology, In press.

[32] E. Calabrese, "Bowel ultrasound for the assessment of Crohn's disease," Gastroenterology \& Hepatology, vol. 7, no. 2, pp. 107109, 2011.

[33] J. Dong, H. Wang, J. Zhao et al., "Ultrasound as a diagnostic tool in detecting active Crohn's disease: a meta-analysis of prospective studies," European Radiology, vol. 24, no. 1, pp. 26-33, 2014. 


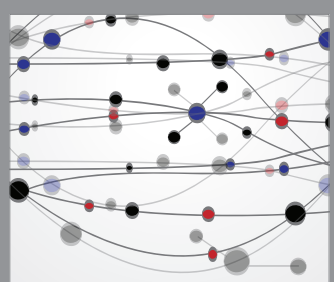

The Scientific World Journal
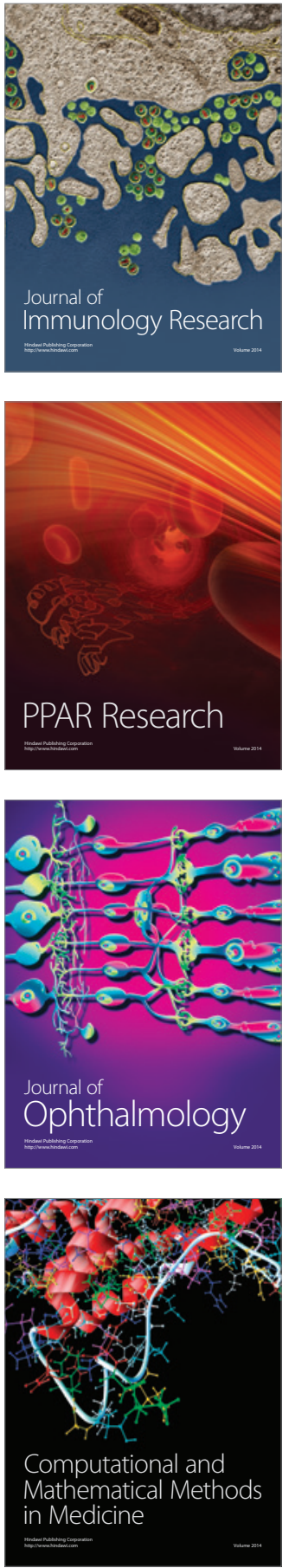

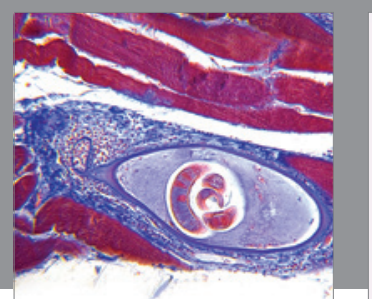

Gastroenterology Research and Practice

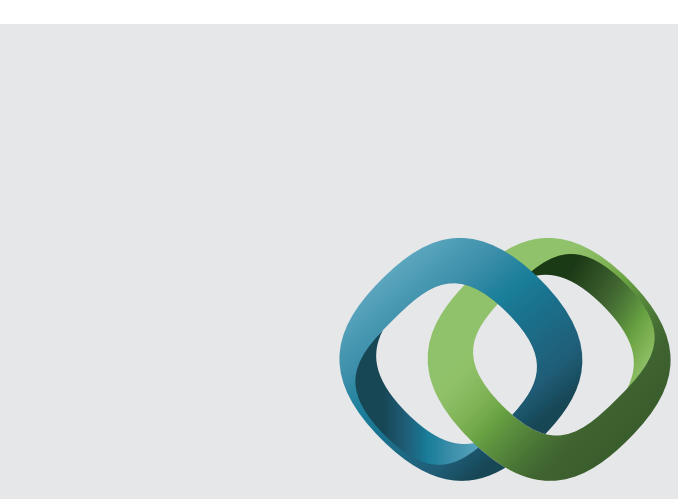

\section{Hindawi}

Submit your manuscripts at

http://www.hindawi.com
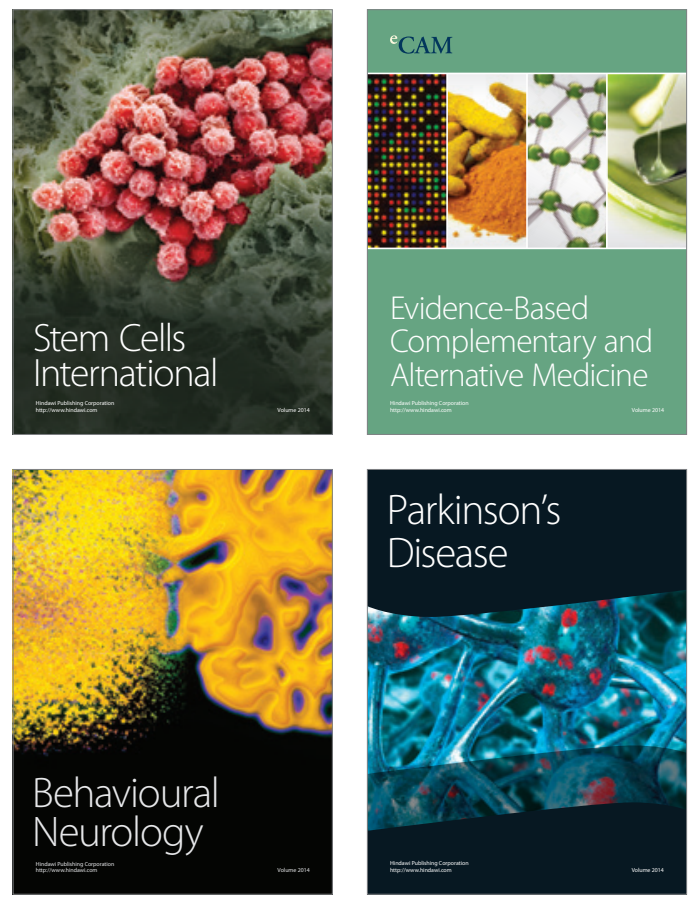
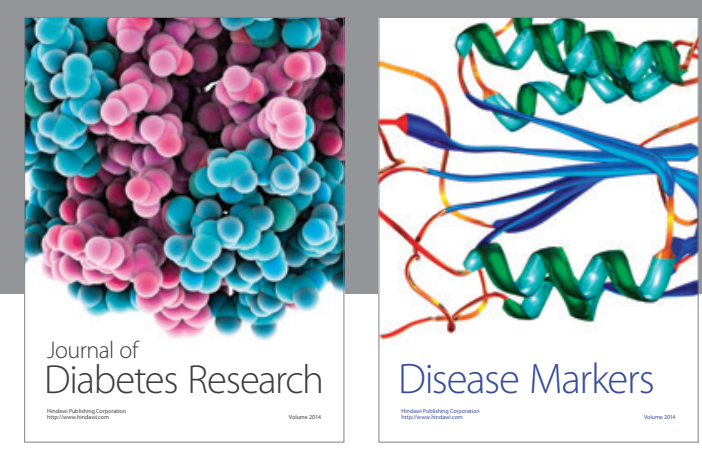

Disease Markers
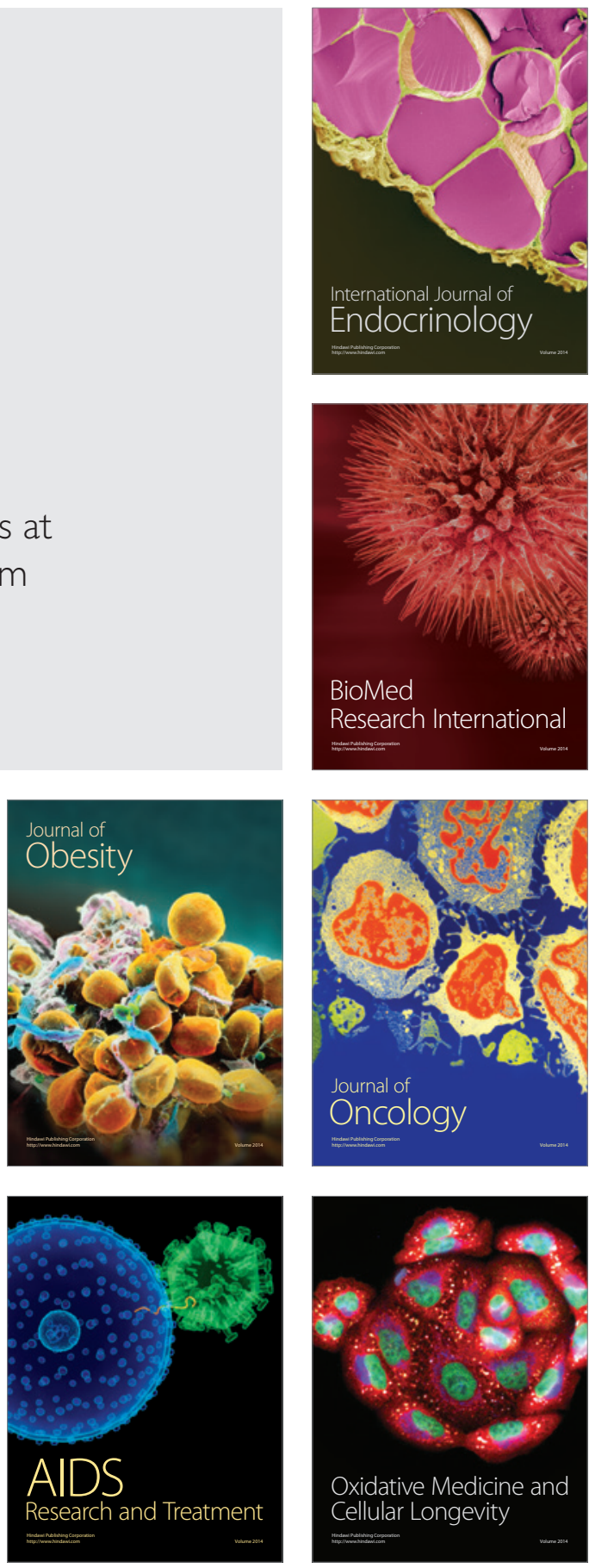